\title{
Transmission Strategy of the Competition -2017 FIBA $3 X 3$ U18 Basketball World Cup
}

\author{
Guowei Sun \\ Department of Journalism(Sports journalism orientated), Chengdu Sport University, Chengdu 610041, China. \\ 605239039@qq.com
}

Keywords: the FIBA 3X3 U18 Basketball World Cup; transmission strategy; media transmission

\begin{abstract}
FIBA 3X3 U18 World Cup has been ended on July 2 in Chengtu. This competition is the first basketball world cup held by China. Although this event has filled the blank in the domestic holding of the international A-level basketball games, the problems that have been exposed by the tournament transmission should be taken seriously. This paper has carried out a concrete study on the transmission strategy of the competition-2017 FIBA 3X3 U18 Basketball World Cup, hoping to bring some enlightenment for the related people in the field.
\end{abstract}

\section{Introduction}

Behind the success of the 2017FIBA3X3 U18 basketball World Cup, the influence of the U18 basketball World Cup has not spread to the whole country, which must be taken seriously by us. Besides, to provide reference for the future holding of various large-scale competitions by combing the relevant experience and lessons, this paper thereupon implements a concrete study on the transmission strategy of the tournament-U18 basketball World Cup.

\section{Overview of 2017 FIBA 3X3 U18 Basketball World Cup}

$3 \times 3$ basketball stems from the street basketball and is more entertaining than the traditional fustal basketball. In addition, it can be closely connected with the urban culture and urban life, which becomes the characteristic of the basketball. It is precisely because the $3 \times 3$ basketball has a series of characteristics that are closely connected with modern city life, so the city government of Chengtu begins to arrange tournaments in 2016. And then the 2016 FIBA 3X3 challenge round in Chengtu, 2017 western 3 X3 basketball champion challenge round and various folk competitions will create a hot atmosphere of 3 X3 basketball competition in Chengtu, which just leads to the successful holding of 2017 FIBA U18 basketball World Cup. Table 1 provides a visual comparison of the three vs three basketball match and the fustal basketball, and we can learn more about this basketball game in combination with the table.

Table 1 Comparison between three vs three basketball and the fustal basketball

\begin{tabular}{|c|c|c|c|c|c|}
\hline $\begin{array}{c}\text { three } \\
\text { basketball }\end{array}$ & VS & three & \begin{tabular}{l}
\multicolumn{2}{c}{ Competition } \\
time: $\quad$ relatively \\
shorter
\end{tabular} & $\begin{array}{l}\text { Attack-defence space: } \\
\text { big }\end{array}$ & $\begin{array}{c}\text { Defending } \\
\text { function:limited }\end{array}$ \\
\hline $\begin{array}{l}\text { the } \\
\text { basketball }\end{array}$ & & fustal & $\begin{array}{l}\text { Competition } \\
\text { time: long }\end{array}$ & $\begin{array}{l}\text { Attack-defence space: } \\
\text { relatively small }\end{array}$ & $\begin{array}{l}\text { Defending } \\
\text { function: relatively } \\
\text { high }\end{array}$ \\
\hline
\end{tabular}




\section{Transmission Strategy of 2017 FIBA 3X3 U18 basketball World Cup}

\section{Publicizing Cultural Characteristics}

Before the holding of U18 basketball World Cup, the city government in Chengtu has already $\mathrm{t}$ successfully built a strong basketball atmosphere in Chengtu through a series of 3X3 basketball events. However, because the basketball atmosphere exists only in Chengtu, there is no real upsurge of $3 \mathrm{X} 3$ basketball all over the country, which has a negative effect on the transmission of the U18 basketball World Cup. To avoid the recurrence of this kind of transmission problems in the future, the publicity of the cultural characteristics of 3x3 basketball must be taken seriously.

$3 \times 3$ basketball has 450 million fans worldwide. And this game itself can merge together organically with music, dance, extreme sports, street art, and e-sports to realize diverse competition forms, which belongs to the main cultural feature of 3x3 basketball. And if this kind of cultural feature can be transformed into a favorable chance for the transmission of U18 basketball World Cup, the national influence of this tournament definitely will be further promoted. Table 2 shows directly the cultural characteristics of three vs three basketball match.

Table 2 Cultural Characteristics of three vs three basketball

\begin{tabular}{ll}
\hline Cultural characteristics & Performance \\
\hline Close to modern urban culture & Realizing diverse tournaments \\
Branch of Hip-hop culture & Organic fusion with music, dance, extreme \\
& sports, street art, and e-sports \\
\hline
\end{tabular}

City government of Chengtu held the international music festival in Du Jiangyan, Chengtu 3x3 basketball tournament, dance camp of international youth street dance contest during the corresponding period of U18 basketball World Cup in 2016, which all belong to the publicity combined with cultural characteristics. Nevertheless, compared with many pure holdings of 3x3 basketball competition, this sort of cultural feature appears to be separated from 3x3 basketball. Thus, the author suggested to realize the isomorphism between culture and transmission when spreading the tournament-U18 basketball World Cup, which can lead to the combination of China's modern urban culture and the $3 \times 3$ basketball. Besides, this can greatly improve the cultural characteristics of the basketball World Cup, and then cause the formation of the unique cultural appeal, which will also bring more positive impact on the transmission of U18 basketball World Cup [1].

\section{Strengthening cooperation with the media}

Apart from the publicity of cultural characteristics, it is essential to strengthen cooperation with the media in order to truly realize the better dissemination of the U18 basketball World Cup. However, in many tournaments of U18 basketball World Cup, the dissatisfaction of many media people has had a negative impact on the transmission of the U18 basketball World Cup. The organizing committee before the competition said to try his best to meet the arrangement of an interview with Yao Ming, which is failed to be realized. Besides, the seats reserved for the media during opening ceremony were commandeered provisionally. All of the above situations have brought negative effects. Although this is connected with the first holding of international A-level basketball league matches for the organizers, the corresponding negative influence of the tournament transmission must cause our attention. Table 3 shows directly the media cooperation problems of U18 basketball World Cup.

Table 3 Media cooperation problems of U18 basketball World Cup

\begin{tabular}{lc}
\hline Media cooperation problems & Performance \\
\hline Interview problems & Failure of realizing the interview with Yao \\
Problems of reserving seats for the media & Ming \\
\hline
\end{tabular}

To really by strengthening cooperation with the media to step up the spread of the World Cup with high quality, the integration of media power and the creation of competitive and high-quality tournament programs can be said the relatively typical and effective means.

1) For the media power of integration, the application of this method is mainly through the all-round communication between the U18 basketball World Cup and the media to realize the 
promotion of the tournament transmission effect. Combining with the features of U18 basketball World Cup and 3x3 basketball, it is not difficult for us to find that TV media and various new media can show directly the features of $3 \times 3$ basketball. Besides, if these features can be flexibly utilized to create the nationwide atmosphere of U18 basketball World Cup , they can bring stronger sense of participation for most people who love basketball. And then $3 \times 3$ basketball can thereby be popularized in our country. The effect and influence of U18 basketball World Cup will greatly be enhanced [2].

2) As for the creation of high-quality competition program, the application of this method needs to be consistent throughout the U18 basketball World Cup. During the warm-up period of the U18 basketball World Cup, if city government of Chengtu, the Chinese basketball association, Sichuan TV station, and Sichuan provincial sports bureau can jointly produce a wonderful TV program with the $3 \times 3$ basketball as the core, and amplify the influence of the TV program to the TV programs such as "Give Me Five", "Running Man" and other alike TV shows through publicity, the influence of the 3 x 3 basketball and U18 basketball World Cup will both be promoted nationwide. The warm-up of the U18 basketball World Cup and the interaction between the official games and TV programs will also promote their mutual influence, and then the transmission effect of the U18 basketball World Cup will also be enhanced greatly. It is worth noting that the relevant programs still continue to be carried out after the end of the competition, the influence realized by 3x3 basketball still be kept and participants in 3x3 basketball are cultivated, which will also bring strong support for the subsequent competitions.

\section{Paying attention to the selection of transmission strategy}

In order to ensure the better transmission effect of U18 basketball World Cup, transmission strategy must also be taken seriously. The transmission strategy mentioned here can be specifically summarized as paying attention to the cooperation with various television stations and application of new media.

\section{Paying attention to the cooperation with many TV stations}

Although the U18 basketball World Cup is hosted by Chengtu, it is obviously not possible to rely on Chengtu and Sichuan TV stations solely to meet the needs of transmitting the U18basketball World Cup. The author thereby suggested to avoid ignoring the cooperation with other television stations as far as possible for the U18 basketball World Cup. Then every level of TV stations can serve for the transmission of the U18 basketball World Cup, so the influence of the U18 basketball World Cup will naturally be promoted greatly nationwide.

\section{Paying attention to the application of new media}

The use of new media can't be ignored in order to ensure that the U18 basketball World Cup will be spread better. The combination of WeChat and Weibo to jointly spread the U18 basketball World Cup competitions in high quality and the rebroadcast by the joint bullet screen websites are both belong to the typical examples of using the new media.

1) For the spread of the U18 basketball World Cup competitions in high quality by combining WeChat and Weibo, the cooperation with WeChat and Weibo belongs to the relatively effective transmission method of the U18 basketball World Cup competitions. And then the full coverage of China's Internet population with the largest population will actually spread the influence of the U18 basketball World Cup to the whole country.

2) As for the rebroadcast of the joint bullet screen websites, even though the influence of the bullet screen websites is far inferior to WeChat and Weibo, they have an unusual influence on the spread of new things and the spread of young people with the leader of bilibili, which has a high degree of corresponding to the main body of the U18 basketball World Cup. And the competition rebroadcast caused by this will also definitely have a more positive impact on improving the influence of the U18 basketball World Cup. Table 4 shows directly the conjunction between the bullet screen websites and 3x3 basketball. 
Table 4 Conjunction between bullet screen websites and 3x3 basketball

\begin{tabular}{ll}
\hline Conjunction & Performance \\
\hline Superposition in front of the crowd & Young people group \\
Curiosity about new things & Group who are curious about new things \\
\hline
\end{tabular}

\section{Conclusion}

This paper studies on the transmission strategy of the tournament-the 2017 FIBA 3x3 U18 basketball World Cup. And the author discusses the three kinds of transmission strategies of the U18 basketball World Cup in detail as follows: publicizing the cultural features, strengthening the cooperation with the media and paying attention to the selection of transmission strategy. This paper aims at bringing certain inspiration for professionals by means of the above methods.

\section{References}

[1] Opel A. Communication, Media, and American Society[J]. Journalism \& Mass Communication Quarterly, 2004, 81(1):199-200.

[2] Frasca R. Communication, Media, and Identity: A Christian Theory of Communication[J]. Journalism \& Mass Communication Quarterly, 2007, 84(2):397-399. 